\title{
The Application of Web Services in the Monitoring System of Internet of Things GUO Qiong ${ }^{1, a}$ \\ ${ }^{1,2}$ Nanyang Medical College, Nanyang 473000, China \\ a GuoQiong2014@yeah.net
}

Keywords: Web Services, Monitoring System, Internet of Things

\begin{abstract}
For large monitoring objects, often require tens of thousands of concurrent report data monitoring sensor, and often involve the high-speed data acquisition frequency, resulting in high speed large concurrent real-time transmission of data flow, the design of the monitoring system of communication service becomes the system critical modules, communication service module performance to a large extent determines the overall performance of the monitoring system, also Internet monitoring system of high real-time requirements. In this article, through the Web services in the application of Internet monitoring system, realized the monitoring system of heterogeneous communication platform, shielding the underlying data, different types, different operating and the difference between heterogeneous platforms, has a certain universal applicability.
\end{abstract}

\section{Introduction}

The Internet of things is through the radio frequency identification (RFID), infrared sensors, global positioning system (GPS), such as the laser scanner information sensing device, according to the contract agreement, to any items in the form of a cable or wireless and Internet connection, such as computing, storage, handling a concerned things of static and dynamic information knowledge network, to realize intelligent identification, location, tracking and monitoring and management of a network [1]. At present has been widely used in intelligent home, remote medical treatment, industrial automation, environmental monitoring, military applications, smart grid, and other fields.

With the rapid development of Internet technology in recent years, more and more Internet technology have been put into practical use. Using Internet technology monitoring is a new kind of effective method of safety regulation. For large-scale monitoring objects, often require tens of thousands of concurrent report data monitoring sensor, and often involve the high-speed data acquisition frequency, resulting in high speed large concurrent real-time transmission of data flow, the design of the monitoring system of communication service became is very important to the whole system module, can communication service module largely determines the performance of the overall performance of the monitoring system, also the Internet monitoring system of high real-time requirements [2]. Web service technology can build cross-platform, loose coupling and strong interactivity, the advantage of application system, suitable for solving the above problems. Therefore, this paper proposes a Web services used in the solution of Internet monitoring system, points out the applying Web services to feasibility and advantage of Internet monitoring system.

\section{Web Service technology}

With the continuous development of Web technology, through the Internet or Intranet access various database has been very easy. But may encounter similar to the following situation: when you want to access a MIS data, only to find that can't use the Internet or the database is not open, at this point is impossible to realize the query operation, and the emergence of the Web Service provides the possibility. Business functionality through Web Service, create the client application invokes the Web Service, make the business functions and the corresponding data into the client, make the customers have a copy directly from the server program and data, can be realized in the network disconnected local data access operations [3]. 
Essentially, Web services are commonly used protocol through the discrete software components to interact with other software components. Remote clients and applications using the SOAP protocol based on XM L via the HTTP protocol, access to the Web Service. The final Web Service architecture is shown in figure 1.

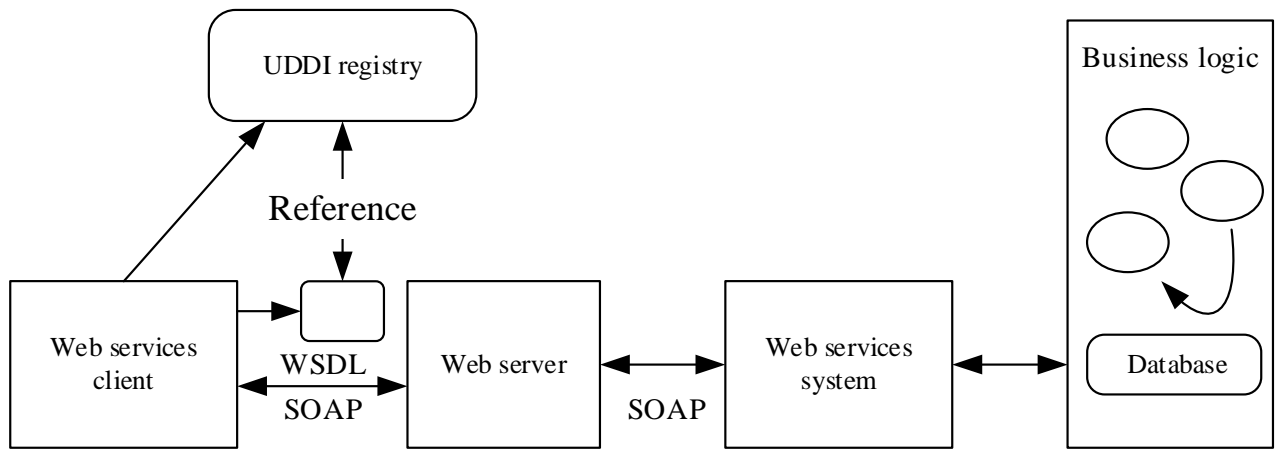

Figure 1. Architecture of Web Service

Due to the use of XML and SOAP, access services, independently of the implementation scheme, therefore, is both Web Service component architecture and the advantages of the Web. Similar to the component development process, Web Service has two characteristics: (1) it has a lookup Service optional registration; (2) it has public interface with clients to invoke the service. In addition, the Web Service also has is better than that of some characteristics of the development of components, such as the use of standard Web protocol to communicate; it can be accessed through the Web; Support is not coupled loose coupling between distributed systems, etc. Web Service has 2 main platforms: the.net and J2EE. The.net platform successfully meet the requirement of Web Service scalable, safe, efficient and portable data and code requirements.

\section{Internet of Things monitoring system research}

Internet of Things usually can be summarized as three domains: perception layer, is usually based on the technique of physics, chemistry, biology and so on all kinds of sensors to collect data; The transport layer, its mainly divided into two kinds of wired and wireless transmission mode; The application layer, to gather up data for intelligent classification, query processing and decision support, etc. Internet monitoring system for communication service is an important part of the Internet of the transport layer [4-5]. For Internet of Things monitoring system, its basic structure can be described as shown in figure 2.

Perception layer. Things networking perception layer is positioned on the first layer of the three layer architecture technology. Is the role of perception layer physical real world and virtual world of information, with the aid of a temperature sensor, a two-dimension code tag, camera, a variety of GPS terminal sensing equipment acquisition perception at the bottom of the information, and these physical information into digital information, transfer to the platform.

Network layer. The Internet network layer is located on the two floor. The network layer after layer functions to establish network aware layer and bridge, all kinds of information networking application layer problems responsible for selling things networking perception layer collected, and the information accurately and efficiently delivered to the application layer of the Internet of things. The network layer is based on modern mobile communication technology and Internet technology, through a variety of wireless data transmission equipment.

Application layer. The IOT application layer in the third layer. The application layer is to create things, wide interface, realize the intelligent application of the Internet of things. Function integrated system application layer at the bottom, through the development of the software, to provide a friendly interactive interface for users, and can realize the networking application of operators. Based on IOT application layer industry including green agriculture, industrial monitoring and control, city management, remote medical treatment. 


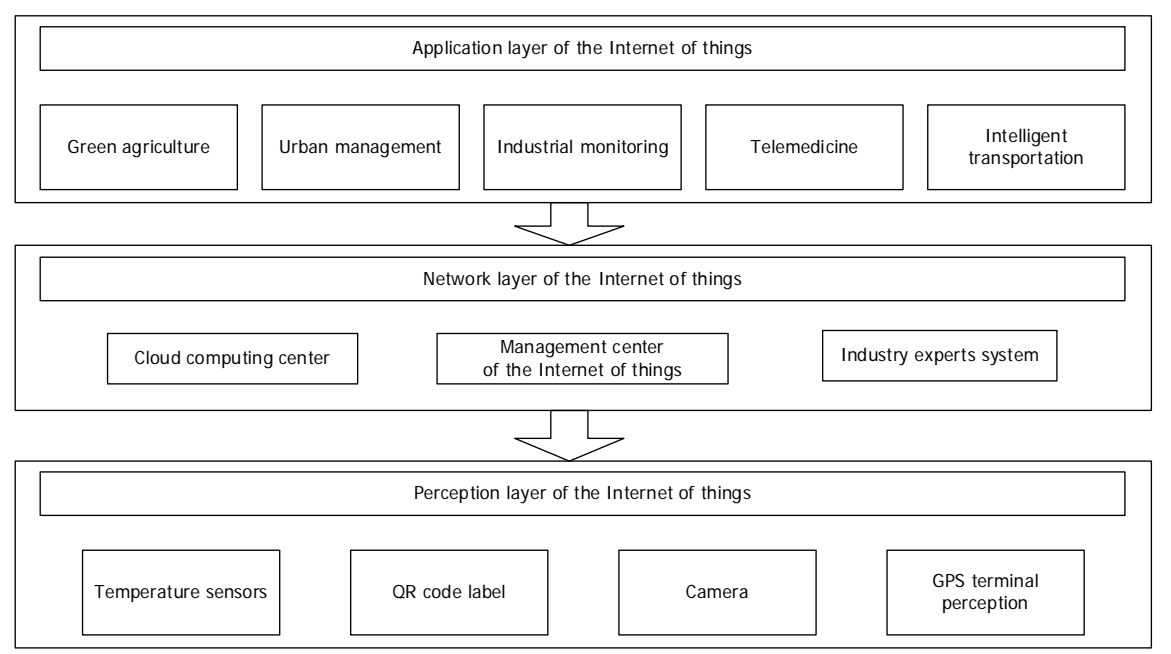

Figure 2.Technology architecture of Internet of Things monitoring system

\section{Workflow of Internet of Things monitoring system based on Web service}

Internet of Things monitoring system based on Web service is a standard, architecture based on good and have good extensibility of software system, and based on the Internet of middleware, to adapt to the Internet of things changing needs, and to ensure that the use of data exchange system application system can run normally and to keep its autonomy. Platform of the embedded data acquisition terminal host to gSOAP agreement release data, thus to realize the data collection and the independence of the underlying equipment, makes the equipment more transparent to the application server to transmit data. Basic sharing platform is based on SOA architecture technology, so as to improve the versatility of the platform, using XML as a data transfer standard, easily achieve a variety of sources of data conversion to each other. Due to the loosely coupled Web Services, using the Web Service mechanism, to achieve transparent access to data, among the upper application of enterprise's database to interconnection between heterogeneous systems. The Internet of Things monitoring system based on Web service workflow is shown in figure 3.

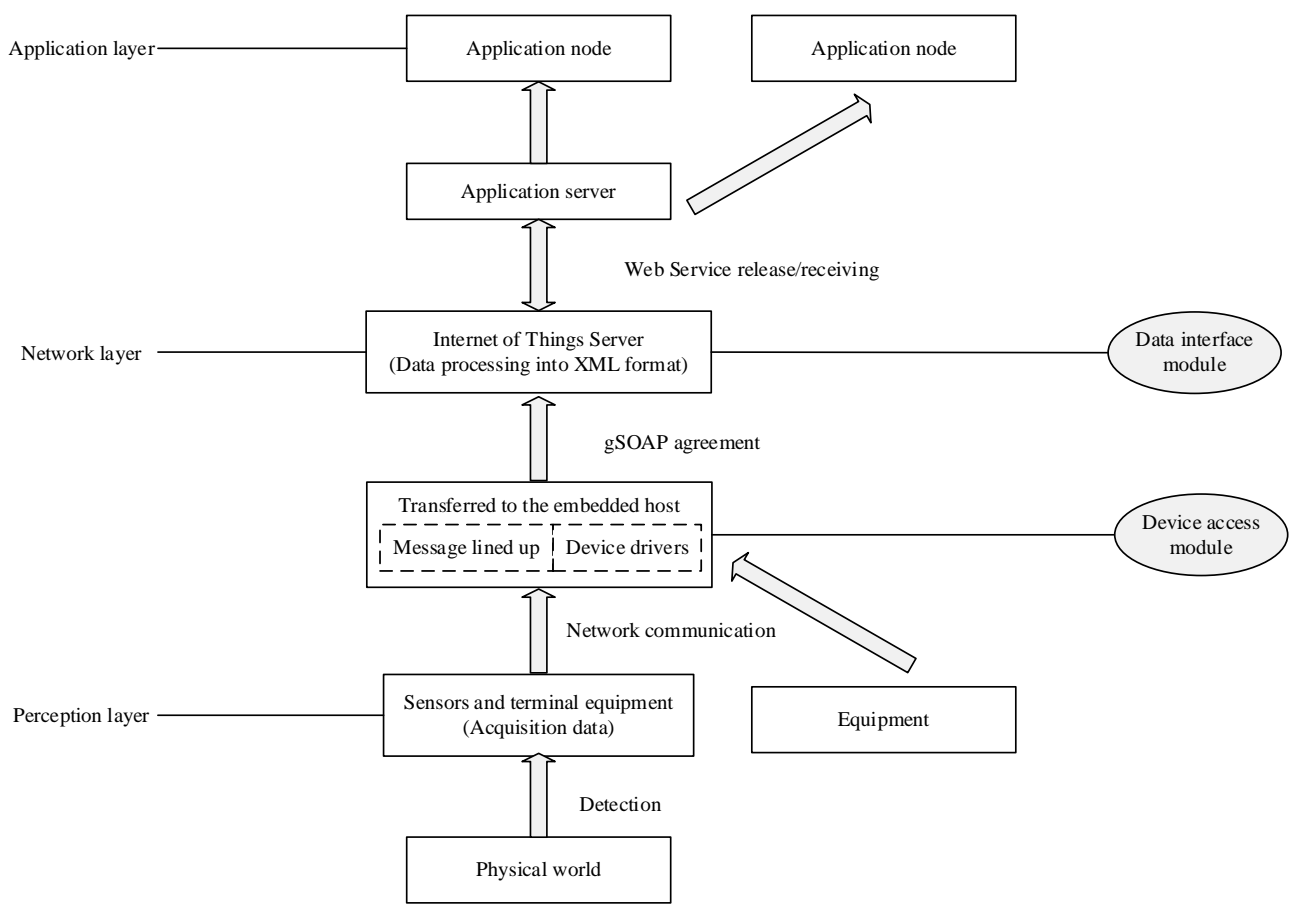

Figure 3.The workflow of Internet of Things monitoring system based on Web service 
Equipment acquisition data. Through the sensor acquisition data outside of human world and the physical world, such as digital temperature/humidity sensor to collect the temperature/humidity data.

Embedded host data access equipment. Communication through the network, sensor network technology to transmit data to the installation of the host of the embedded operating system.

Release data by gSOAP agreement. Embedded host collected data transmission by means of gSOAP agreement publish a Web service to the Internet server 'thus to realize the data acquisition of the underlying device independence, makes the equipment more transparent to the application server to transmit data.

Internet of Things server release data. Service to the application Service implements network based data interaction module handle data into XML form, and then through the form of a Web Service, the data published to the application server Service.

The management of Internet of Things server application server. The application server to provide service for possessions are connected to the server management functions. All application nodes on the application server operation, can obtain the corresponding data. Server receives the Internet server from new or updated data services, through the message mechanism to notify theme application nodes have subscribed to the service.

Node service request or response. Application node grading the needed data services, the application server will be to deal with all the request message application node. Platform in accordance with the local situation of the theme of the subscription application node distribution or routing process, the data application node release to subscribe to this topic.

\section{Acknowledgements}

Science and technology of Nanyang 2013GG053.

\section{Conclusion}

Internet of things with the computer, the Internet and the world three times in the wave of information industry, and its comprehensive application of the current different technology, multiple disciplines, got a lot of countries, enterprises and research institutions and research. With the deepening of China's informatization process, to speed up, transportation, agriculture, the environment in many fields such as Internet monitoring system, the specialty of Internet of things technology. In this article, through the Web services in the application of Internet monitoring system, realized the monitoring system of heterogeneous communication platform, shielding the underlying data, different types, different operating and the difference between heterogeneous platforms, has a certain universal applicability.

\section{Reference}

[1] L. Atzori, A. Lera, and G. Morabito: Computer networks, Vol.54 (2012) No.15, p.2787

[2] J. Luo, Y. Chen, and K. Tang: International Conference on Future (Sanya, China, Dec13 - 14, 2009), Vol. 1, p.482

[3] W. Baoyun: Journal of Electronic Measurement and Instrument, Vol.23 (2011) No.12, p.1

[4] E. Welbourn, L. Battle, and G. Cole: Internet Computing, Vol.13 (2010) No.3, p.48

[5] A.C. Sarma, J. Girão J: Wireless Personal Communications, Vol.49 (2011) No.3, p. 353 